\title{
An IT strategy development framework for small and medium enterprises
}

\author{
Andreas Drechsler \\ School of Information Management, Victoria University of Wellington, Wellington, New Zealand \\ University of Duisburg-Essen, Essen, Germany
}

Stefan Weißschädel

Albellus Software AG, Duisburg, Germany

Paper submitted to and accepted by the Information Systems and E-Business Management journal. The final publication is available at Springer via http://dx.doi.org/10.1007/s10257-017-0342-2/

\begin{abstract}
We design and evaluate an IT strategy development framework for small and medium enterprises (SMEs). The framework's objective is to provide a theoretically grounded, empirically validated, uncomplicated, and accessible framework to develop an IT strategy for an SME. The framework is a social artifact whose purpose is to guide the design of two other artifacts: an IT strategy process and an IT strategy plan. We rely on design science research, combined with action research, to design, apply, evaluate, and refine the framework in a specific SME in the sales industry. People responsible for managing the IT in an SME can use the framework to design or refine their IT strategy, in order to make better-informed IT strategy decisions, to improve the utilization of their SME's usually scarce IT resources, and ultimately increase their IT's business value contribution. We also gain an enhanced understanding of IT strategies' role in SMEs and provide methodological implications for social artifact design.
\end{abstract}

\section{Introduction}

The intentional definition and execution of an IT strategy are widely regarded as key instruments for the efficient and value-creating use of information systems for business purposes (Earl 1989; Ward and Peppard 2002). Following Chen et al. (2010, p. 235), who provide an extensive and integrative IT strategy literature review, we understand IT strategy as "as the organizational perspective on the investment in, deployment, use, and management of information systems." While Chen et al. (2010) use the term IS strategy, we use IT strategy to avoid confusion with a common IT strategy element in the literature. There, IS strategy only covers the actual applications-related strategy as part of a comprehensive IT strategy (cf. Section 2.3).

A more in-depth look at Chen et al.'s (2010) findings reveals that the majority of papers cover the development and application of IT strategies in large organizations. Owing to the complexities of IS use and management in such organizations, an elaborate IT strategy can provide a much needed orientation and a systematic approach to manage the multitude of existing IT management challenges. Further, such an undertaking is worthy of special IT strategy research attention. To a greater or lesser degree, the IS/IT management of small and medium enterprises (SMEs) often faces goals and challenges similar to those that large enterprises face, even though they provide fewer IT services and have fewer IT infrastructure elements. Similarly, if SMEs' business models, or their internal processes, are reliant on information systems, their IS' efficiency and value-creation are no less important to them than for large enterprises. While the criteria for classifying an enterprise as a small or medium one vary by country (Australian Government 2014; European Commission 2014; U. S. Census Bureau 2014), staff size is a common denominator. We therefore define a small business as having fewer than 20 employees, and a medium business as having fewer than 250 employees. However, SMEs' typical dearth of resources (Welsh and White 1982), together with the much smaller organizational scale, presents additional challenges in the IT strategy context: The smaller number of specialized IT personnel and executives means fewer resources to execute management tasks. In addition, the smaller personnel pool also means less tolerance for management overheads in the form of elaborate management processes and instruments, such as the development and execution of elaborate IT strategies.

The global strategy consulting firm AMI Partners published a study (AMI Partners 2013) that provides evidence of these conceptual deliberations. They found that, in the surveyed countries (US, Australia, India, Brazil, and Chile), a vast majority of small and medium enterprises do not have an internal IT department, but that 30 to 40 
percent have their most tech-savvy employee manage their IT as an involuntary IT manager (IITM). These employees do not necessarily have a specific IT education or training, and also have to cover their normal business functions. The study estimates that these small firms annually lose about USD 24 billion in business productivity by using IITMs instead of a separate, internal IT department with dedicated, formally assigned, and appropriately trained IT staff. A total of 26 percent of IITMs report they believe they are not qualified to manage the IT. Six out of ten IITMs would like to simplify their company's technology solutions to alleviate their day-to-day IT management.

This simplification of the existing IT infrastructure can be set in motion by developing a suitable IT strategy. An appropriately formulated IT strategy will not only provide a coherent foundation for executing the necessary steps, but will also help ensure that the simplified IT infrastructure still fulfils the business needs. An IT strategy can also serve as a foundation for the possible introduction of a dedicated IT management position within an SME. However, the literature on IT strategy (Earl 1989; Ward and Peppard 2002) often assumes that such positions exist, that IT organizations are large, and therefore mentions a large number of aspects that should be considered in an IT strategy. Even literature that specifically focuses on the SME context often proposes planning processes that IITMs might not be willing, or even able, to execute alone. For instance, Levy and Powell (2000) and Duhan (2007) propose two approaches to develop a partial IT strategy for SMEs (mainly covering the IS strategy, cf. Section 2.3). As we will show in greater detail in Section 2.4, utilizing these approaches for effective IT strategy development in SMEs required external interventions due to the approaches' comprehensiveness. Once applied, it remains questionable whether these approaches will lead to a sustained strategic impact as soon as there are no more resources for external interventions. We therefore conclude that the current IT strategy literature does not suit the SME context well. To our knowledge, the IS discipline has not as yet risen to the challenge of providing a suitable and, simultaneously, uncomplicated and accessible framework for developing a suitable IT strategy for SMEs.

This paper's main practical contribution is therefore the development and evaluation of such an IT strategy development framework for SMEs. From a design science research perspective, the IT strategy development framework is a social artifact for a managerial audience. Since the artifact's purpose is to guide an actual IT strategy development, it is actually a social meta-artifact, i.e. an artifact whose purpose is to guide other artifacts' development (Iivari 2003). Our corresponding theoretical contribution is threefold: First, we position our social meta-artifact - the IT strategy development framework - as generally applicable to SME contexts beyond our single evaluation context. It is therefore not a situated, but an abstract or mid-range artifact (Gregor and Hevner 2013). We appropriate extant knowledge of IT strategies and their development and create a novel IT strategy development artifact tailored to the SME context; our contribution therefore comprises a so-called type VIII research contribution (Mandviwalla 2015). Second, we provide several implications regarding social meta-artifact design in general and social artifact design for a managerial audience in the SME context. Third, we highlight that the IT strategy literature often assumes that a strategic understanding of IT already exists within an organization. We show that, in an SME context, there may not be such an understanding and illustrate how our framework can help produce such an understanding.

The remainder of the paper is structured as follows: In the second section, we cover the theoretical and conceptual foundations of IT strategies and IT strategy development within and outside the SME context. In the third section, we outline the research methodology we employed to design, evaluate, and refine the framework. In the fourth section, we describe the framework's application in a practical setting and its subsequent refinement. In the fifth section, we discuss the framework's main contributions to the IT strategy and the design science literature, and its implications for practice. In section six we discuss the limitations of our research. In the seventh and last section, we provide a summary and suggest avenues for further research, as well as for the framework's future applications.

\section{Theoretical and conceptual foundations}

\subsection{The role and importance of IT and IT strategy for SME competitiveness}

In the past, SMEs have been characterized as only engaging in strategic planning activities to a limited extent (Sexton and Van Auken 1985). Lyles et al. (1993) recommend that SMEs take up formalized strategic planning 
processes to achieve an increased competitive advantage. Maranto-Vargas and Gomez-Tagle Rangel (2007) confirm that technology contributes to the development of competitive advantages - 'soft' technology such as organizational processes, procedures, and technical knowledge more so than actual 'hard' technology. Here, IT strategies serve to combine the technical knowledge of the firm's IT with business knowledge to create actionable plans to increase the IT's business value contribution. Singh et al. (2008) identify IT applications as one of 15 areas in which SMEs can develop a strategy to increase their competitiveness. In addition, we agree with Carr's (2003) basic notion - despite the criticism directed at his methodology - that enterprises (including SMEs) should treat the IT infrastructure underlying the IT applications as a commodity, unless there are clear indications that emergent innovative hardware technologies - such as mobile computing in the past - have a significant potential to provide competitive advantages. Likewise, Proudlock (1999) advises SMEs to treat IT applications as a commodity and to buy market-leading off-the-shelf solutions, in order to minimize the implementation risk.

However, to actually achieve increased competitiveness, a continuous fit (Bergeron et al. 2001) or alignment (Venkatraman et al. 1993) is needed between the IT applications and the infrastructure on the one hand and the business strategy and the business processes on the other hand. Here, the IT strategy serves either as the link between the business strategy and the IT to continuously re-align the latter, or even as an enabler of business transformation. While mainly directed at and analyzed in large firms, this relation was found to also be true for SMEs (Cragg et al. 2002; Olugbode et al. 2007). Factors affecting the extent of IT business alignment in SMEs are: the communication and partnership between business and IT managers, personnel skills, assessments of IT investments' value contributions, the organizational scope and architecture, and the SME owner's knowledge of IT (Gutierrez et al. 2009; Chao and Chandra 2012).

Combining these research findings with the findings from the AMI Partners (2013) study cited in the Introduction, it becomes obvious that a lack of attention to IT strategies and associated IT strategy processes can indeed compromise SMEs' competitiveness, and therefore requires research attention.

\subsection{Conceptualizations of IT strategy and its relation to business strategy}

Following Chen et al. (2010, p. 235), we understand IT strategy as "as the organizational perspective on the investment in, deployment, use, and management of information systems." Chen et al. (2010) have developed the framework depicted in Figure 1, which is based on current management and IT strategy literature, to summarize the various theoretical perspectives on IT strategies. Note that we use the terms IT, IT role, and IT strategy instead of IS, IS role, and IS strategy to avoid confusion with a common IT strategy element in the literature (cf. Section 2.3).

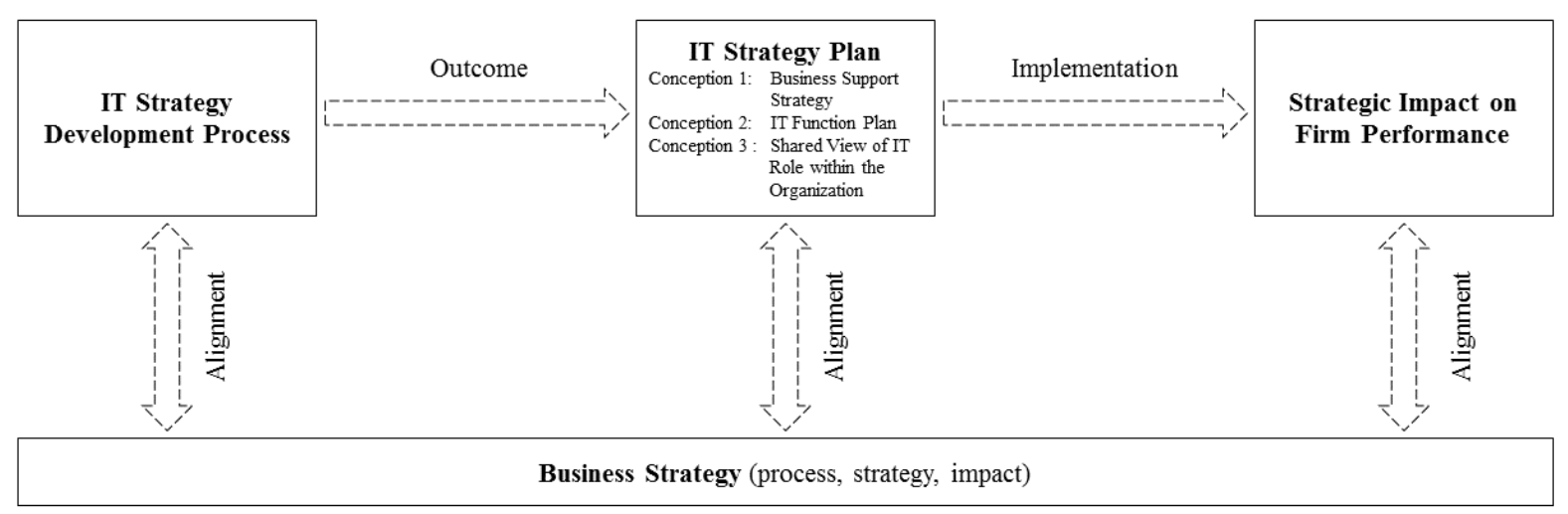

Fig 1. Conceptual framework for IT strategy (Chen et al. 2010, modified)

First of all, Chen et al. (2010) distinguish between a process and a content perspective on IT strategies. The output of the IT strategy development process determines the content, i.e. the eventual IT strategy plan. The IT 
strategy plan's implementation then impacts the information systems, their use in an organization, and ultimately, the firm performance. All three elements (process, plan, and impact) need to be considered in the business and IT strategy alignment context.

Chen et al. (2010) further distinguish three conceptual IT strategy perspectives: 1) the IT strategy as the use of IT to support the business strategy, 2) the IT strategy as the master plan for the IT function, and 3) the IT strategy as the shared view of the IT role within an organization. In all perspectives, an IT strategy's desired impact is to increase the long-term firm performance or competitive advantage. In perspective 1), the IT strategy is clearly subordinate to the business strategy and the desired impact is achieved by supporting the business strategy implementation. In the perspective 2), the business strategy is not explicitly considered and the desired impact is achieved through effective IT asset acquisition and allocation. In perspective 3), an integral perspective is taken on the business and the IT strategy development. Here, the desired impact is achieved by gaining a shared understanding between the business and the IT to guide subsequent IT decisions. While perspectives 1) and 2) are polar opposites in terms of their emphases (business-centric or IT-centric), perspective 3) is a higher-order concept representing the top management's (comprising the business and IT executives') joint perspective on the IT role.

In their framework, Chen et al. (2010) focus on the IT strategy content and the associated impact, treating the process element as the context. Here, Näsi (1999) provides a broader and complementary perspective on IT strategy development. He identifies the strategic management process, strategic game-playing, and strategic leadership as three modes of strategy-making.

In respect of the strategic management process, Näsi (1999) describes a high-level process model consisting of five steps as a commonly used process model for strategy development in enterprises. These steps are: 1) defining the mission, 2) analyzing the firm and its environment, 3) formulating the strategy, 4) implementing the strategy, and 5) evaluating and controlling the performance.

In the strategic game-playing mode, Näsi (1999) sees strategies as an instrument to position an enterprise as a player with certain current, or possible, products on the market landscape, which other players populate simultaneously. This mode means that an IT organization deliberately positions itself in a specific way (e.g., in terms of alignment or governance) toward the business and in the way it thinks about its delivered IT products and services. Thus, positioning and repositioning through strategic game-playing comprise a strategy impact that cannot directly be measured in monetary value and can contribute to achieving Chen et al.'s (2010) shared understanding perspective mentioned above.

Finally, the strategic leadership mode emphasizes the role and the traits of the person(s) executing the strategy process. Näsi (1999) highlights that it is important for a strategic planner to have various leadership traits, such as professional skills (being assertive, visionary, and able to set agendas), interpersonal skills (building networks, coping with cultures and coaching), and personal skills (self-development, being a person of power, and being totally dedicated). The underlying assertion is that the better developed the traits, the better the IT strategy planner can provide effective strategic leadership. In turn, this strategic leadership effectiveness directly affects the actual extent of an IT strategy's impact, i.e. the "arrows" in Figure 1.

\subsection{Common content elements of IT strategy plans}

As clarified in Figure 1, the IT strategy plan is the key foundation for the IT strategy implementation that, in turn, leads to the IT strategy's actual impact. According to Earl's (1989) and Ward and Peppard's (2002) seminal works on IT strategies, an IT strategy plan should cover three areas: the information management strategy (IM strategy), the information and communication technology strategy (ICT strategy), and the information systems strategy (IS strategy). Figure 2 provides a detailed illustration of the three areas of an IT strategy plan and their link to the overall business strategy. 


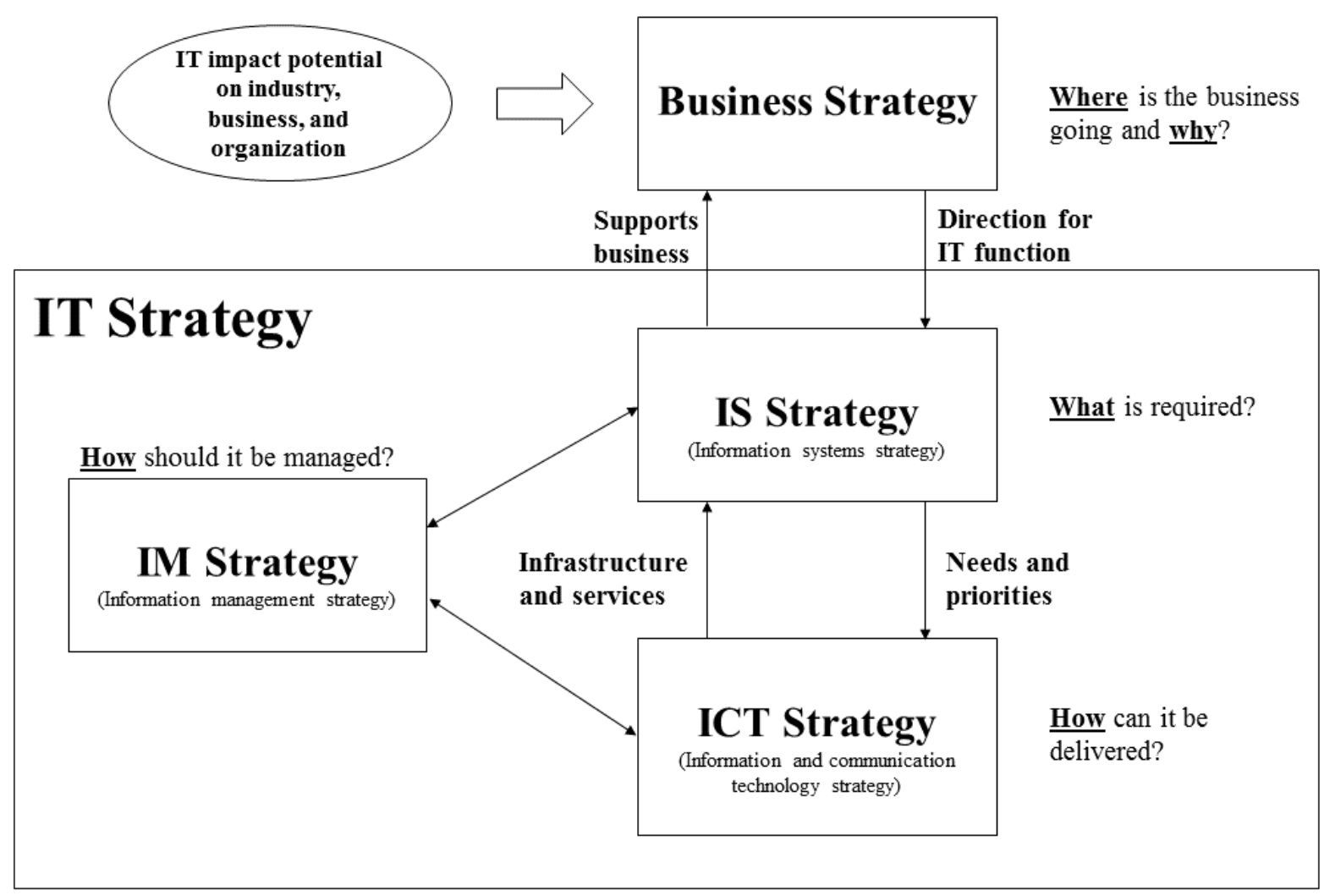

Fig. 2 Commonly mentioned IT strategy areas and their connection to the business strategy as summarized by Hofmann and Schmidt (2007)

The IM strategy contains the IT organization's future processes, structure, and resources, the methods and instruments for managing this strategy, as well as the IT organization's targeted future position and role in the company. The ICT strategy covers the organization's technological infrastructure and its operation. The IS strategy deals with the provision of applications to fulfill the business strategy needs within the organization. Accordingly, the IS strategy's main purpose is to determine the demand for business applications and to plan, develop, and provide IT applications to meet this demand. All three strategies influence each other: The IM strategy determines the resources (personnel, processes, tools) to introduce, change, operate, and maintain the applications (which the IS strategy defines) and the infrastructure (which the ICT strategy defines).

In respect of actual IT strategy development, there are numerous suggestions regarding the specific elements that need to be considered in each of the three areas - not only in IS research literature, but also in practitioner-oriented works. For example, Earl (1989), as well as Ward and Peppard (2002), does not only distinguish between the different IT strategy areas, but also provides extensive guidance on how these areas should be covered in an actual IT strategy plan. However, following their guidance would lead to an extensive framework for IT strategy development. Moreover, we expect that an extensive review of other guidance would expand the framework even further. Owing to SMEs' application context, we do not believe that such a comprehensive IT strategy plan would be regarded as useful. Before we proceed further, we therefore review the extant literature on IT strategy in SMEs to examine the existing approaches to handling the conflict between the prescribed complexity and the SME business reality's need for simplicity. 


\subsection{IT strategy in SMEs}

Compared to the general IT strategy literature, we found that the literature dealing with IT strategy in SMEs excluding papers merely covering IT strategy in the IT alignment context - is scarce and fragmented. The majority of papers with an immediate relevance to IT strategy in SMEs take a conceptual or explanatory stance, providing voluntary or involuntary IT managers in SMEs with comparably little actionable advice. Many papers on IT strategy in SMEs limit their analyses to specific industry sectors or countries. In addition, many frequently cited papers are between 10 and 15 years old, sometimes even older, and do not necessarily reflect current IT management practices in SMEs, or the current state of IT management and IT strategy research.

More recently, Cragg et al. (2013) analyzed the IT management sophistication in SMEs. Based on four traditional management functions (planning, organizing, controlling, leading), they have discovered that, in SMEs, IT planning and IT leading play a more crucial role than the other two. This not only reinforces this paper's planning focus, but also Näsi's (1999) emphasis on the importance of leadership traits for strategic IT planning (cf. Section 2.2). To support strategic IT planning, Levy et al. (1999) classify existing planning frameworks into three framework types that SMEs can use to support their IT strategy planning: awareness frameworks, opportunity frameworks, and positioning frameworks. However, neither type covers the actual IT strategy; they just support the planning process. Levy et al. (2001) also develop a specific framework to assess the information systems' strategic role within an SME in a 2x2 matrix, depending on the customer dominance (low or high) and the strategic focus (IT as cost-inducing necessity or to provide added value). Again, such a framework can aid strategic IT planning, but does not lead to developing a comprehensive IT strategy, which is why we do not present further framework details. Bradshaw et al. (2012) analyze the extent to which SMEs rely on external support to manage their IT in the form of IS consultants. These authors point out that SMEs often miss important opportunities to develop in-house IT and IT management competencies.

For actual information systems strategy planning in SMEs, Levy and Powell (2000) propose a concrete approach and evaluate it in over 40 SMEs in the UK, where the approach was employed by external analysts guided by the method developers. Likewise, Duhan (2007) proposes an actionable toolkit for strategic information systems planning in SMEs. He evaluates the toolkit through participant observation (for instance, as a consultant or a non-executive director) in three cases. However, both approaches only concentrate on an IT strategy subset: the IS strategy and its link to business strategy to develop supportive information systems and organizational capabilities, respectively. Both approaches also rely heavily on other frameworks and instruments for strategic planning. In particular, Levy and Powell's (2000) approach is comprised of more than ten individual management instruments, such as business scorecards, SWOT analyses, and strategic grids. In a similar vein, Duhan's (2007) approach proposes dedicated competence and capability analysis and development models as part of the strategy development process. Taken in sum, both approaches do not conform to the need for simplicity in a typical SME business reality outside of dedicated one-time interventions by researchers or external analysts. While such interventions may certainly have a valuable one-time strategic impact for the SMEs in question, they simultaneously lack sustainability. Moreover, neither approach covers the IM and the ICT strategy areas. The literature contains other approaches (Bleistein et al. 2006a; Bleistein et al. 2006b; Samavi et al. 2008) to formalize parts of strategy and IT strategy development through social modeling (e.g., to model business goals and models and to derive system-level requirements from business goals). While these approaches promise a more comprehensive and systematic approach to IT strategy development and IT alignment, they are also on a level of sophistication that is at odds with the need for simplicity in SMEs that may not even have dedicated IT staff and executives.

With regard to comprehensive perspectives on IT strategy development in SMEs, the practitioner-oriented literature offers numerous proposals regarding the elements an IT strategy plan should at least contain. However, we found it difficult to assess these proposals' quality ex-ante, as they contain different subsets of the comprehensive IT strategy plan areas (IM strategy, IS strategy, ICT strategy, cf. Figure 2 and Section 2.3). Therefore, we used the publication outlet as a proxy to assess the proposals' quality. We regarded a publication in an established series (Handbook of Business Strategy) published by a well-known publisher in academia (Emerald) to be superior to proposals published in trade journals due to higher review and inclusion standards. In this publication, Savin (2004) suggests the following minimal elements for an IT strategy plan: 1) the existing applications, 2 ) the development of new or additional applications, 3) the IT infrastructure, 4) the maintenance, 5) the IT operations, and 6) the IT security. To assess comprehensiveness, we mapped these six IT strategy plan elements on 
the three IT strategy areas (IS strategy, IM strategy, ICT strategy). The result shows that the majority of the elements (existing applications, development of new or additional applications, IT infrastructure, maintenance, IT security) correspond to the ICT and IS strategy's elements and "IT operations" and - to a certain extent - "IT security" (policies, etc.) fit under the IM strategy umbrella. Hence, Savin's (2004) six elements cover all three IT strategy areas while, simultaneously, addressing the need for simplicity in an SME context. However, Savin (2004) deems these six IT strategy plan elements to be the minimal ones, but does not explicate the grounds for selection - thus providing little assurance in terms of coherence and soundness of selection.

Overall, the current state of the research, as well as the practitioner-oriented literature on IT strategy for SMEs, provides little coherent, up-to-date, and actionable guidance on how to approach the challenge of formulating an IT strategy in an SME.

\section{Research methodology}

In this section, we describe design science research as our main underlying research paradigm, the corresponding design and evaluation methodology, the artifacts we want to design in detail to address the identified need for IT strategy development in SMEs, and the setting in which we evaluate and refine the artifacts.

\subsection{Design science research to develop and evaluate the IT strategy development framework as a social meta-artifact}

In a research context, the search for a solution for an identified and yet unsolved real-world problem in the IS discipline - such as the lack of effective and uncomplicated IT strategies in SMEs - calls for an IS design science research (DSR) approach (Peffers et al. 2007). Design science research calls all designed (artificial) objects that address a real-world problem artifacts (Simon 1996). Ideally, the artifact development is to be grounded in theory. Artifact utility is the main "dependent variable" that design science researchers need to evaluate to assess the artifacts' effects in practice (Hevner et al. 2004; Gregor and Hevner 2013; Gill and Hevner 2013). Gill and Hevner (2013) further advise considering artifact fitness aspects in terms of "reproduction" (artifact adaptability to foster the artifact's adoption in varying contexts) and "survival" (artifact adaptability on the instance level to sustain the artifact's utility over time).

Iivari (2015) distinguishes two basic strategies to conduct design science research: Either to design an abstract / conceptual meta-artifact that addresses a relevant real-world problem class and to instantiate the artifact in specific problem contexts later on (strategy 1), or to design a specific solution for a specific client in a specific context and to only worry about generalization during the project (strategy 2). Meta-artifacts are artifacts that support other artifacts' development. In particular, Iivari (2003) distinguishes two IT meta-artifact types: product and process artifacts. The former are technical IT system components, constructs, models, and methods that contribute to specific IT system instantiations. The latter comprise information system development methods through which the product artifacts are developed. Since we are not concerned with IT system components, but IT strategies, the meta-artifact we set out to design is social in nature.

In order to achieve research outcomes of managerial relevance, Nicolai and Seidl (2010) recommend focusing on research that is conceptually relevant - i.e., is suited to inspire and guide managerial action - and does not aim to be instrumentally relevant, i.e. is intended to be immediately applicable as-is. These authors' rationale is that the unavoidably abstract research outcomes of management research (an IT strategy is certainly managerial and not technical) cannot take the specifics of each particular application context into account; it is up to the managers to adapt and tailor conceptually relevant research outcomes to their situation. Consequently, a meaningful research outcome of this paper should be one that - in the words of Pandza and Thorpe (2010) - triggers a path-dependent (in case of an existing IT strategy), or even a path-creating (if there is no IT strategy at all), change process within an SME.

Ideally, at the end of this change process, the SME's voluntary or involuntary strategic IT manager will have created and executed an IT strategy that helps achieve the overall business goals and that he or she can maintain. Such a view also corresponds to Iivari's (1986) suggestion to evaluate information systems development methods in terms how well they support the free and informed choices of the involved stakeholders. It further mirrors 
Iivari and Maansari's (1998) understandings of information systems development methods as a resource to support action (in our case, strategic IT planning), or as a reminder (a "shopping list") of the specific tasks the action entails. The key difference in both cases is that we are not interested in developing information systems, but IT strategies.

Since we do not believe that any generalized IT strategy would be applicable (and thus, relevant) to a significant number of SMEs, our goal is to design an IT strategy development framework for SMEs that can successfully trigger path-dependent, or path-creating, change processes and guide the responsible strategic IT manager to develop an impactful IT strategy. From a more abstract perspective, such an IT strategy development framework is a social meta-artifact, or an IT meta-strategy, comprising IT strategy content and process elements.

\subsection{Artifacts, design objectives, and evaluation setting}

Since our framework is a social artifact within the IT management domain, we follow an IS DSR methodology which integrates the current state of social, or management-oriented, design science research with the IS design science research discourse specifically tailored to design IT management artifacts (Drechsler 2013). Based on the works of van Aken $(2004 ; 2005)$, the methodology not only proposes designing the artifact of interest - in our case, the IT strategy development framework for SMEs - but also the means to introduce it in its application contexts. In our case, these contexts encompass the SMEs that might want to use the framework in the future. Drechsler's (2013) methodology calls the former artifact the object design and the latter artifact the implementation design. We ground our framework's initial design - the object design - in the existing literature on IT strategies for SMEs. Specifically, we select those elements of the extant descriptive and prescriptive - actionable knowledge (Gregor and Hevner 2013) of strategies and IT strategies that fit the SME context.

Since our object design - the IT strategy development framework - is not only a social artifact, but also a social meta-artifact with a purpose to guide the responsible strategic IT manager to develop an impactful IT strategy (see previous section), we need to employ a differentiated view on its effects.

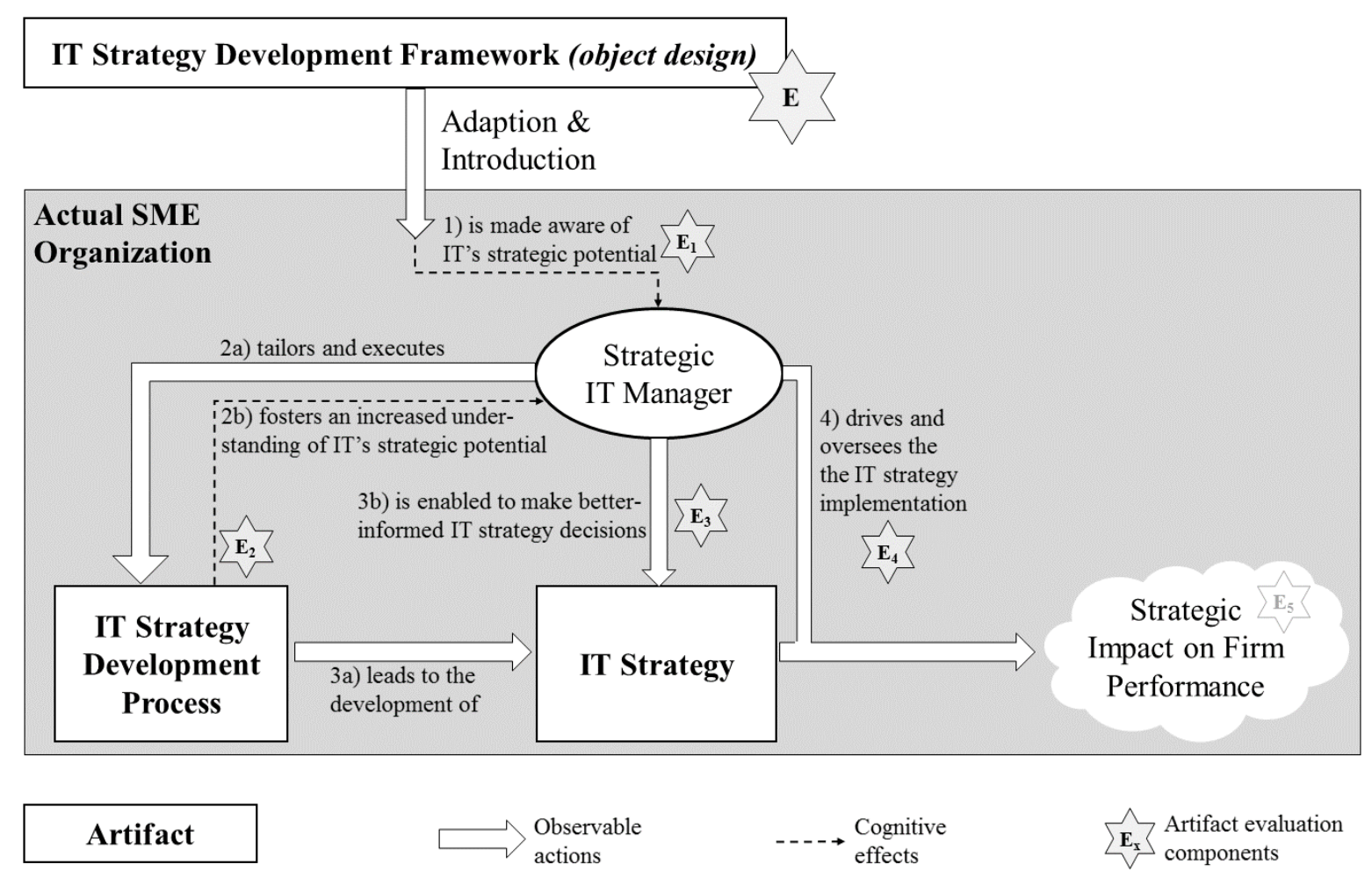

Fig. 3 Role of our framework in IT strategy development and evaluation components 
Figure 3 extends Chen et al. (2010)'s IT strategy conceptualization (cf. Figure 1) by incorporating the prominent role the SMEs' strategic IT manager plays in utilizing our framework. The dashed arrows represent different facets of the framework's conceptual relevance for the SME's strategic IT manager that contribute his or her cognitive development to make better-informed IT strategy decisions, while the block arrows (carried over from Figure 1 and partially extended) represent the more observable actions that take place during the IT strategy development and execution.

First, the framework serves the initial purpose of making a voluntary or involuntary strategic IT manager aware (or increasingly aware) of IT's strategic potential for their SME (arrow 1). Afterwards (arrow 2a), he or she executes the IT strategy development process (the first social artifact generated by using the framework). By doing so, he or she gains an even further increased understanding of IT's strategic potential (arrow 2b). Executing the IT strategy development process leads to the development of the actual IT strategy plan (the second social artifact, arrow 3a). During this process, the previously increased awareness and understanding of IT's strategic potential leads to better-informed IT strategy decisions that are ultimately documented in the resulting IT strategy plan (arrow 3b). Subsequently, the strategic IT manager implements the IT strategy (arrow 4), which in turn leads to the actual strategic impact on firm performance. Figure 3 thus highlights the interplay between the strategic management process and strategic leadership modes of strategy-making (Näsi 1999).

Note that, compared to Figure 1, we changed the graphical representation of the strategic impact on firm performance to a cloud since there are many other factors - that are not part of Figure 3 - within an organization and its dynamic environment that affect the actual and measurable strategic impact of an IT strategy development and implementation on firm performance. Note further that our effective IT strategy conceptualization in Figure 3 does not match any of Chen et al. (2010)'s three IT strategy conceptualizations (business support strategy, IT master plan, shared view of the IT role, cf. Section 2.2). It is not solely a business support strategy, as our IT strategy understanding comprises the IS as well as the IM and ICT sub-strategies (cf. Section 2.3). It does not correspond to a master plan for the IT function, since IT's strategic business potential is emphasized. While the "shared view of the IT role" conceptualization comes closest, it is not directly applicable to many SMEs as they often lack dedicated IT directors or executives. We therefore conceptualize IT strategy for SMEs as follows: For our purposes and context, the IT strategy development serves the purpose of achieving and documenting a sufficient IT understanding on the top management (director) level of SMEs to make well-informed IT strategy decisions and execute them effectively.

To evaluate our framework's overall effectiveness (evaluation component E in Figure 3), we therefore need to evaluate the framework's effects on the IT director's high-level awareness (evaluation component $\mathrm{E}_{1}$ ) and more detailed understanding of IT's strategic potential $\left(\mathrm{E}_{2}\right)$ as well as his or her subsequent abilities to make informed IT strategy decisions $\left(E_{3}\right)$ and to drive the IT strategy implementation $\left(E_{4}\right)$. In an ideal world, we would also measure the actual effect the developed and implemented IT strategy subsequently has on firm performance. However, we regard it as unrealistic to isolate the effects of the IT strategy development from many other internal and external forces as noted in the paragraph above (the evaluation component $\mathrm{E}_{5}$ is therefore kept in gray in Figure 3). In Nicolai and Seidl's (2010) terms, $E_{1}$ and $E_{2}$ correspond to conceptual relevance (the framework's effects on awareness and understanding), while $\mathrm{E}_{3}$ and $\mathrm{E}_{4}$ correspond to instrumental relevance (the framework's effects on decision-making and action-taking).

Against this backdrop, we can now formulate our first design objectives (DOs) for the IT strategy development framework. $\mathrm{DO}_{1}$ and $\mathrm{DO}_{2}$ operationalize artifact utility, split between the two relevance types as discussed above. Note that we use the term operationalize analogously to its use in empirical research - to turn an abstract concept or construct into more concrete terms to make it workable for the subsequent research (even if we do not apply quantitative measures).

$\mathrm{DO}_{1}$ : Effectiveness of the IT strategy development framework to increase a strategic IT manager's awareness and understanding of IT's strategic potential for their SME before and during an IT strategy development process (evaluation components $E_{1}$ and $E_{2}$ in Figure 3).

$\mathrm{DO}_{2}$ : Effectiveness of the IT strategy development framework to increase a strategic IT manager's ability to make well-informed IT strategy decisions $\left(E_{3}\right)$ for and to implement the resulting IT strategy $\left(E_{4}\right)$ in their $S M E$. 
We also need to operationalize artifact fitness - i.e. the artifact's adaptability to foster the artifact's initial adoption in varying contexts as well as its sustainability over time - further, since Gill and Hevner (2013) mainly use the fitness term as an analogy from biology and provide little design-oriented advice on how to achieve either fitness type for IS artefacts.

When we perceive our framework as an innovation and draw on Rogers' (2003) diffusion theory, the two attributes compatibility (to the innovators' needs, values, and beliefs) and complexity stand out as the ones most aligned to the fitness analogy, while Rogers' relative advantage (2003) attribute corresponds closely to the artifact utility discussed above. If our artifact's audience perceives the framework to be too complex, it will not meet their needs and values, and they will therefore not adopt the framework or utilize it sustainably (e.g., yearly) without external intervention. For instance, there is evidence from the project management area that SMEs do not accept complex project management frameworks (O'Connor and Coleman 2009). A major reason these authors found is that the framework users (project managers and members) perceive these frameworks to be overly costly. In addition, we assume that SME IT managers only have very limited resources to invest in IT strategy development and therefore value (and a have need for) simplicity. If the framework is too simplistic, however, it may have only a limited cognitive impact informing and guiding the strategic IT manager's IT strategy decisions, and therefore would not meet their needs either. Subsequently, the framework would have only a limited impact on firm performance, and therefore ultimately a low utility. Lastly, as SMEs come in various sizes and rely on IT to different extents, fitness for our IT strategy development framework needs to consider flexibility as well. Flexibility means that framework users can adjust the necessary or feasible level of detail to their specific contexts' requirements and circumstances. Flexibility is therefore a counter-balance against too little complexity and too much complexity. We therefore formulate our third design objective as follows:

$\mathrm{DO}_{3}$ : Flexibility of the framework's elements to achieve adequacy for a specific application context (i.e. balancing complexity and simplicity), in order to meet the respective strategic IT manager's needs and values as prerequisites for the framework's effectiveness.

Subsequently we designed a generic framework that those responsible for managing an SME's IT can use to develop an initial IT strategy, or refine an existing one. In particular, we considered the following parts of the IT strategy development framework as the object design:

- $\quad$ the three possible modes of IT strategy-making: the strategic management process, strategic game-playing, and strategic leadership (Näsi 1999, cf. Section 2.2)

- the IT strategy conception: business support strategy, master plan for the IT function, and the company's overall IT strategy (Chen et al. 2010, cf. Section 2.2)

- the elements of an actual IT strategy plan: initially Savin's (2004) six elements (cf. Section 2.4), later updated to IS, IM, and ICT strategy as major sections with additional subsections (cf. Section 2.3)

- $\quad$ the IT strategy process (Näsi 1999, cf. Section 2.2)

- $\quad$ the skills the IT strategy planner(s) should have (Näsi 1999, cf. Section 2.2)

We evaluated and refined the framework during several rigor, design, and relevance cycles (Hevner 2007) in the context of a single, yet not untypical, small SME. The SME in which we applied and evaluated our framework is a small sales industry enterprise that sells its partner organizations' IT products. There are two directors (one responsible for, among other things, the IT-related decisions), two full-time employees, and up to four temporary employees. The IT infrastructure's actual maintenance was outsourced to an external IT service provider. Given the distinction between voluntary and involuntary IT manager discussed in the Introduction, the director responsible for IT-related decisions resembles an involuntary IT manager as IT management is not his field of expertise. Simultaneously - as one of two directors - there is no superior that forced him to take on this role; therefore the distinction between voluntary / involuntary IT manager does not quite fit our scenario. Instead, our SME has no dedicated strategic or operational IT personnel at all.

Our rationale for our design and evaluation approach and context choice was threefold: First, we chose this small enterprise for the framework's first application to show its viability even in an enterprise of this size, without a true IT executive, no formalized IT management structures or processes, and no extant IT strategy. Our case thus represents an extreme - yet not untypical - case not yet covered in the literature. Second, a single application context allows a more exploratory research mode. As we could not draw on previous experiences in the literature, we were open to any impact and feedback from our application context and could incorporate the lessons 
learned throughout the cycles in this singular context into our framework design, without the need to coordinate the redesigns with the framework's application in other contexts. Third, we posit that if the framework were to receive a positive evaluation, larger SMEs - with more personnel and more IT applications and infrastructure would benefit even more from an IT strategy formulated by means of the framework's application. We regard extending the framework to subsequent larger application contexts as easier than having to reduce the elements of a previously positively evaluated framework when it is unclear which elements (if any) to remove.

Owing to the exploratory nature of our research, we also did not specify an initial implementation design. Instead, this paper's second author was directly involved in the framework's actual introduction and evaluation processes, and conducted on-site interventions (van Aken 2007) over several cycles. In particular, the framework's introduction in a target organization constituted an organizational intervention comprising interviews, analyses, workshops, presentations, and discussions with the intent to establish an IT strategy that the organization can follow. Thus, our overall research approach is a hybrid between design science and action research, with the action research part replacing the aforementioned implementation design. However, based on our intervention activities, we propose an initial implementation design for our IT strategy development framework during our final cycle (Figure 8). Organizations wishing to utilize the framework in the future can draw on this implementation design.

Note that our approach differs as a design and action research hybrid from Sein et al.'s (2011) action design research (ADR) approach. The major difference between the ADR approach and our approach is that the ADR aims to design emergent information systems artifacts for a particular context and only considers possible generalizations afterwards (similarly to Iivari's (2015) strategy 2 mentioned in Section 3.1), while we intend to develop a generic framework as an artifact for deliberate strategy-making in - potentially - any SME.

In Figure 4, we summarize the contents of the object design and the implementation design and their roles in the scope of our research (limited to a single organization) and beyond (when using the IT strategy development framework in other organizations in the future). In Figures 5 and 6, we show more concrete versions of Figure 4 in two research cycles, while Figure 8 contains a concrete proposal for the implementation design based on our action research experiences. 
IT Strategy Development Framework for SMEs (object design)

\begin{tabular}{|c|c|}
\hline \multicolumn{2}{|c|}{ Modes of IT strategy-making (Näsi 1999) } \\
\hline Planner Skills (Näsi 1999) & IT Strategy Conception (Chen et al. 2010) \\
\hline $\begin{array}{c}\text { IT Strategy Development Process Template } \\
\text { (Näsi 1999) }\end{array}$ & $\begin{array}{l}\text { IT Strategy Plan Template } \\
\text { (cf. Sections } 2.3 \text { and } 2.4 \text { ) }\end{array}$ \\
\hline
\end{tabular}

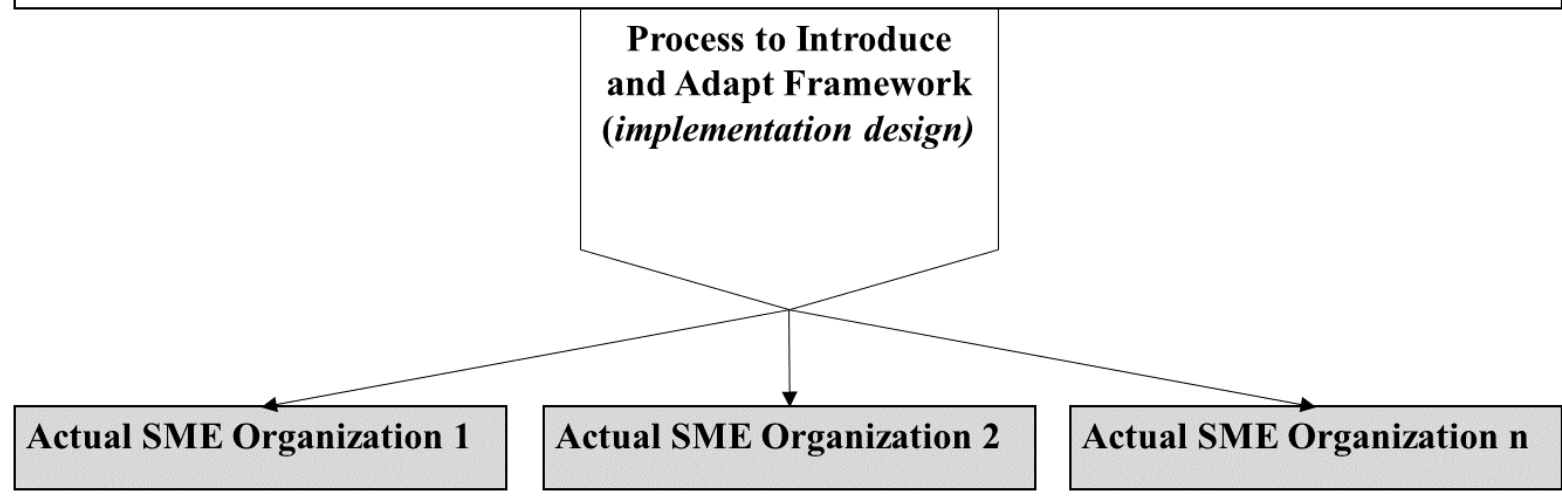

Fig. 4 Content and roles of our object design (the IT strategy development framework) and the corresponding implementation design in the research process

The corresponding evaluation activities are closely related to the intervention activities of each research cycle and aim to evaluate how well the framework achieves the three design objectives formulated above. Note that the second author's 'embeddedness' in the application context throughout the entire project does not allow us to clearly separate intervention, data collection, evaluation, and redesign activities. In each cycle, these activities comprised observing and analyzing the as-is situation in the application context, instantiating the generic IT strategy development framework to the context's particulars (including preparing and tailoring actual IT strategy document templates), briefing and interviewing the director responsible for IT, discussing the elements to maintain and areas for the framework's future improvement, and writing a summary and an action / modification plan for the next cycle.

Initially, we planned to conduct semi-structured interviews to systematically evaluate how well the design objectives were reached, while simultaneously being open to explore the positive and the negative impacts, effects, and issues with the respective framework state and the actual IT strategy beyond the prepared interview guide. However, based on the rich feedback we received, the interview settings quickly developed into discussion rounds and artifact redesign mini-workshops that rather quickly rendered any prepared guides largely obsolete. Since we wanted to take advantage of the positive dynamics we encountered, we ensured a mutual understanding through a written summary and a written action / modification plan for the next cycle. We could therefore not follow the interview guide as in a traditional question and answer interview setting. In each cycle, the exchange with the artifact user addressed specific and different issues with the framework and the resulting IT strategy identified in the previous cycle (see section 4 for details). These findings formed the input for the subsequent cycle in order to change the framework accordingly and subject the redesigned framework to another research cycle. Note that we were unable to complement our interviews and observations with document analysis (e.g., IT financial performance comparisons or IT architecture models) due to the relevant SME's lack of available formal documents. 


\section{Design and evaluation of the IT strategy development framework}

In this section, we detail the evolution of our IT strategy development framework in a SME over four design cycles.

\subsection{Cycle 1: Initial framework design and artificial ex-ante evaluation}

As discussed in the Introduction, the inefficient use of IT resources in SMEs is the initial class of real-world problems that triggered our design science and action research process. We surmise that, specifically, involuntary IT managers only undertake the measures required to maintain the existing IT infrastructure and do not develop an IT strategy unless their top management directs them to do so. This inhibits a controlled and efficient investment in, as well as the deployment, use, and management of, information systems. Therefore, our research goal was to develop an IT strategy development framework that fulfils the three DOs specified in Section 3.2 and ultimately allows voluntary and involuntary strategic SME IT managers to quickly and easily formulate an initial IT strategy plan and execute it.

Figure 4 contains the framework's basic elements that needed to be instantiated for the context at hand. For the IT strategy plan template, we rejected the prevailing body of prescriptive knowledge of an IT strategy plan's elements (cf. Section 2.3), as it does not conform to the need for simplicity as specified in $\mathrm{DO}_{3}$. Levy and Powell's (2000) and Duhan's (2007) SME-specific approaches to plan a subset of a comprehensive IT strategy - the IS strategy - also do not conform to the need for simplicity, as they rely heavily on other elaborate planning frameworks and instruments. Instead, we used Savin's (2004) proposed minimal requirements for the IT strategy plan template (cf. Section 2.4).

To keep the process as simple as possible for the first iteration, we did not address the other framework elements of Figure 4 in greater detail. However, in order to evaluate their conceptual relevance of how well they are suited to achieve a cognitive impact on the IT director in-line with $\mathrm{DO}_{1}$, we added them to the discussion points we covered during the introduction process (cf. Figure 5). In particular, we used Näsi's (1999) three modes of IT strategy-making, his five-step IT strategy process, and the skills Näsi (1999) deems important for an IT strategy planner (cf. Section 2.2). Likewise, we considered Chen's (2010) three IT strategy conceptions: a business support strategy, a master plan for the IT function, and a shared view of the IT role in the company (cf. Section 2.2). We did not specify distinct steps for the implementation design in the first introduction process, but used action research instead (cf. Section 3.2).

Figure 5 visualizes the initial framework. Note that this figure just summarizes and synthesizes the respective framework elements. Preparing appropriate document templates (for the IT strategy plan), or other appropriate means of triggering a meaningful exchange (for the discussion points) during the actual organizational intervention (cf. Section 3.2), is a key task to implement the framework. 


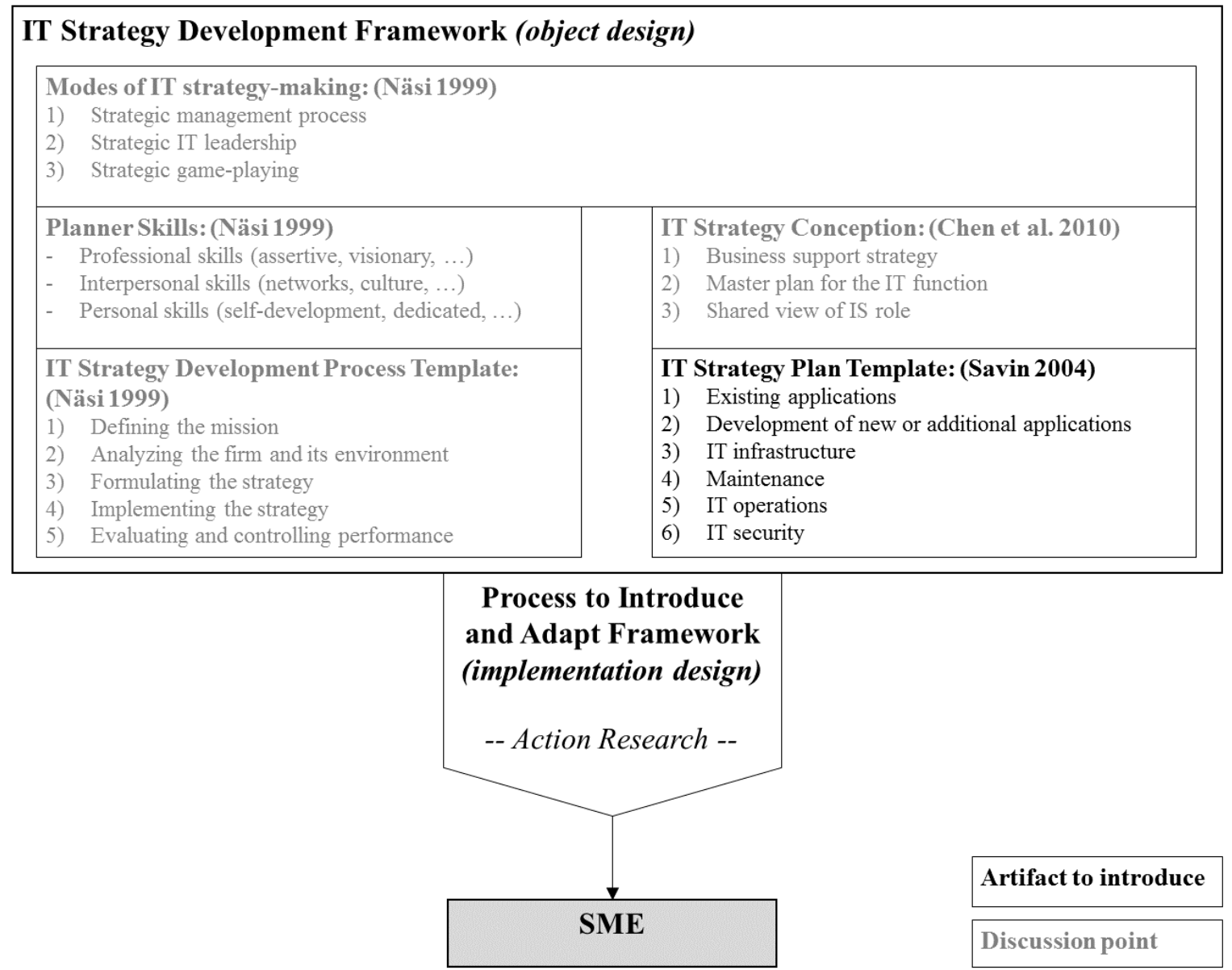

Fig. 5 The initial IT strategy development framework

To conclude this first cycle, we conducted an ex-ante evaluation of the IT strategy development framework in an artificial setting (Pries-Heje et al. 2008). We viewed it through the lens of a future user - a voluntary or involuntary IT manager in an SME - wanting to introduce a new IT strategy. First, there is a low number of elements (six) to consider for the IT strategy plan. Second, most of the elements concern the tangible IT systems and infrastructure. We regarded both aspects as positive for the framework's utility and fitness. Regarding $\mathrm{DO}_{1}$, a low number of elements to consider corresponds to a low barrier to increasing one's understanding about IT's strategic potential. It will also be comparably easy to draw concrete links between more "hands-on" IT strategy elements and their corresponding strategic business potential. Regarding $\mathrm{DO}_{2}$, the low number of elements makes it easy to develop and implement an IT strategy plan from the ground up. Regarding $\mathrm{DO}_{3}$, the low number of elements fulfils the future SME framework users' simplicity needs. The emphasis on tangible IT elements further corresponds to the SME framework users valuing the IT strategy-making's leadership mode over any formalized management processes and instruments that an IM strategy would specify. However, the initial framework lacks flexibility in case the framework is found lacking comprehensiveness.

\subsection{Cycle 2: Introducing and evaluating the initial framework}

On the basis of our ex-ante evaluation, we did not change the framework before its actual introduction in a specific SME (cf. Section 3.2). The simple context allowed us to focus on the IT strategy development framework (the object design), because, as an action research setting, the implementation design consisted of the initial adaption of the IT strategy plan template (first redesign) and consulting sessions with the director responsible for the IT (second redesign). 
In order to fill out the IT strategy plan template (cf. Figure 5), we needed to first develop supporting documents reflecting the current as-is state of the processes, the IT infrastructure, and applications in the SME. During the workshop sessions, it became apparent that the template's elements indeed provide useful guidance for directing the IT strategy development. Simultaneously, there is no guidance for assessing whether the resulting IT strategy plan satisfactorily covers the template's elements. Further, we also found that the proposed IT strategy framework lacks a prescriptive strategic element in a future-oriented perspective. The result was that the director believed that the descriptive element in the initial version was over-emphasized. Nevertheless, he evaluated the developed IT strategy plan as a step in the right direction towards achieving an understanding about the different areas of IT management and use that require, or influence, decision-making.

Regarding Näsi's (1999) three modes of IT strategy-making (cf. Figure 5), the director questioned the relevance of the game-playing perspective. While he indeed emphasized leadership rather than the management processes, he wondered whether this needed to be explicitly mentioned as part of the IT strategy, since this only involved him. In addition, he recognized most of Näsi's (1999) strategy process phases as already in use, although with a possible under-emphasis of the final evaluation and controlling phase. With regard to Chen et al.'s (2010) three IT strategy conceptions (cf. Figure 5), he regarded the IT strategy as a business support strategy as the only option for an SME as small as his. In addition, he suggested adding an introductory and a concluding section to the IT strategy plan template to outline the IT strategy's general purpose and the rationale for each element. He also noted that, in terms of the context, the external requirements - which, for example, relevant laws mandate - were missing. The same applied to the internal, non ICT, or IS-related, aspects, such as the IT personnel's skills.

After the formulated IT strategy plan had been used for about a year, we evaluated it a second time with the same IT director with whom we had previously worked, in order to learn more about its effectiveness. Previously, only the most important tasks required to maintain the SME's IT infrastructure had been performed. IT was merely considered a tool to support the business processes. We identified three reasons for this behavior: 1) the focus on the core business, 2) the lack of knowledge and resources to implement additional IT projects, and 3 ) the outsourcing of the actual IT maintenance, which meant there was no strategic oversight. By utilizing the IT strategy development framework early in 2013 to formulate an actual IT strategy, the SME received 1) a strategy document that described the existing IT resources (hardware and software), 2) the processes required to run the IT systems, and 3) a list of future projects to enhance the business performance. The SME's management gained an improved understanding and transparency about the IT and its processes. The director considered this improved understanding valuable, since, with the help of this knowledge, the management could plan its IT investment more strategically. This specifically included a more long-term perspective and an explicit business orientation. Hence, the "effectiveness" design objectives $\left(\mathrm{DO}_{1}\right.$ and $\left.\mathrm{DO}_{2}\right)$ for the development framework were partially fulfilled as the framework managed to improve the strategic understanding of IT and to trigger the development and execution of an impactful IT strategy. Likewise, we considered the "contextual adequacy" design objective $\left(\mathrm{DO}_{3}\right)$ as partially fulfilled, since the IT strategy development and execution were indeed manageable with a low resource investment and thus matched the director's values. The objectives were only partially fulfilled, however, as the director had identified several missing aspects that would lead to a further increase in his ability to make well-informed IT strategy decisions $\left(\mathrm{DO}_{2}\right)$ and thus better match his needs, though for the price of an increased complexity $\left(\mathrm{DO}_{3}\right)$.

\subsection{Cycle 3: Redesigning the IT strategy development framework and reevaluating the redesign}

Based on the feedback received, we revised the IT strategy development framework as follows:

1. A more comprehensive and systematic consideration of the IT strategy plan elements: Since the director identified a few aspects (the IT personnel's skills, the external requirements) as lacking in the initial elements, we deemed Savin's (2004) list (cf. Section 2.4), which we had used in the first cycle, incomplete and too limited. We therefore expanded the IT strategy plan template to cover all three areas discussed in Section 2.3 in greater detail: the IM strategy, the IS strategy, and the ICT strategy. Simultaneously, we maintained the successfully validated elements and assigned them to their respective area. We also added selected, new elements, which were also regarded as lacking, or of interest, during the evaluation.

2. The addition of an introductory and concluding section: We added an introductory section to describe the purpose and scope of the IT strategy plan template, as well as a high-level strategy process. The former's purpose is to choose between Chen et al.'s (2010) three IT strategy conceptions (cf. Section 2.2) to guide the strategy 
process. The high-level strategy process emphasizes the importance of the actual strategic planning activities in respect of each element of the IT strategy and the evaluation.

3. The addition of an IT vision and mission: To emphasize that the IT strategy's purpose was to support the business strategy, we also added a section on the IT vision and mission, which linked the IM strategy and the business strategy (the context). This was introduced by following the first process element in Näsi's (1999) strategy process ("Defining the mission"). We assumed - for the reasons the director mentioned in the previous phase - that, in the majority of SMEs, IT supports the business and therefore also added this aspect to the IT strategy plan template. The redesign, which occurs during the implementation process, also allows for adapting this mission to a different application context, in which a different mission may be more appropriate.

4. A clear distinction between the as-is and to-be perspectives: Based on the director's feedback that the previous framework, which provided no further guidance, over-emphasized the as-is state - and was thus of little use to him - we subsequently emphasized that it is as important to describe the current as-is state as it is to develop an intended future to-be state for each of the three IT strategy areas.

5. The consideration of the planner's leadership traits: Owing to the confirmed high importance of leadership in the specific case, and since we deemed the case a typical one, we subsequently covered this issue explicitly in the IT strategy development framework. In the first edition, Näsi's (1999) leadership skills (cf. Section 2.2) were merely discussion points that we believed the planner, or the planning committee, should have. We also recommended that planners should seek expert advice if the mentioned skills are not available in their organization.

6. The addition of an executive summary: To cope with the IT strategy plan's extended structure, we added an executive summary, which provides a brief overview of the IT strategy.

7. The addition of a document information section: During the previous cycle, it had been noted that general information about the document and the version number were missing. We therefore added a separate section to provide these.

Figure 6 shows the structure of the refined IT strategy development framework for SMEs and Figure 7 the new expanded template elements of the IT strategy plan. As with Figure 5, these figures have a summarizing and synthesizing purpose for the preparation of the actual organizational intervention. Figure 7's elements, for instance, served as headings for the revised IT strategy plan template document. Unfortunately, we cannot show the actual IT strategy document due to its size (12 pages) and for confidentiality purposes. Readers would also find it difficult to understand as they lack contextual knowledge about this particular SME. 


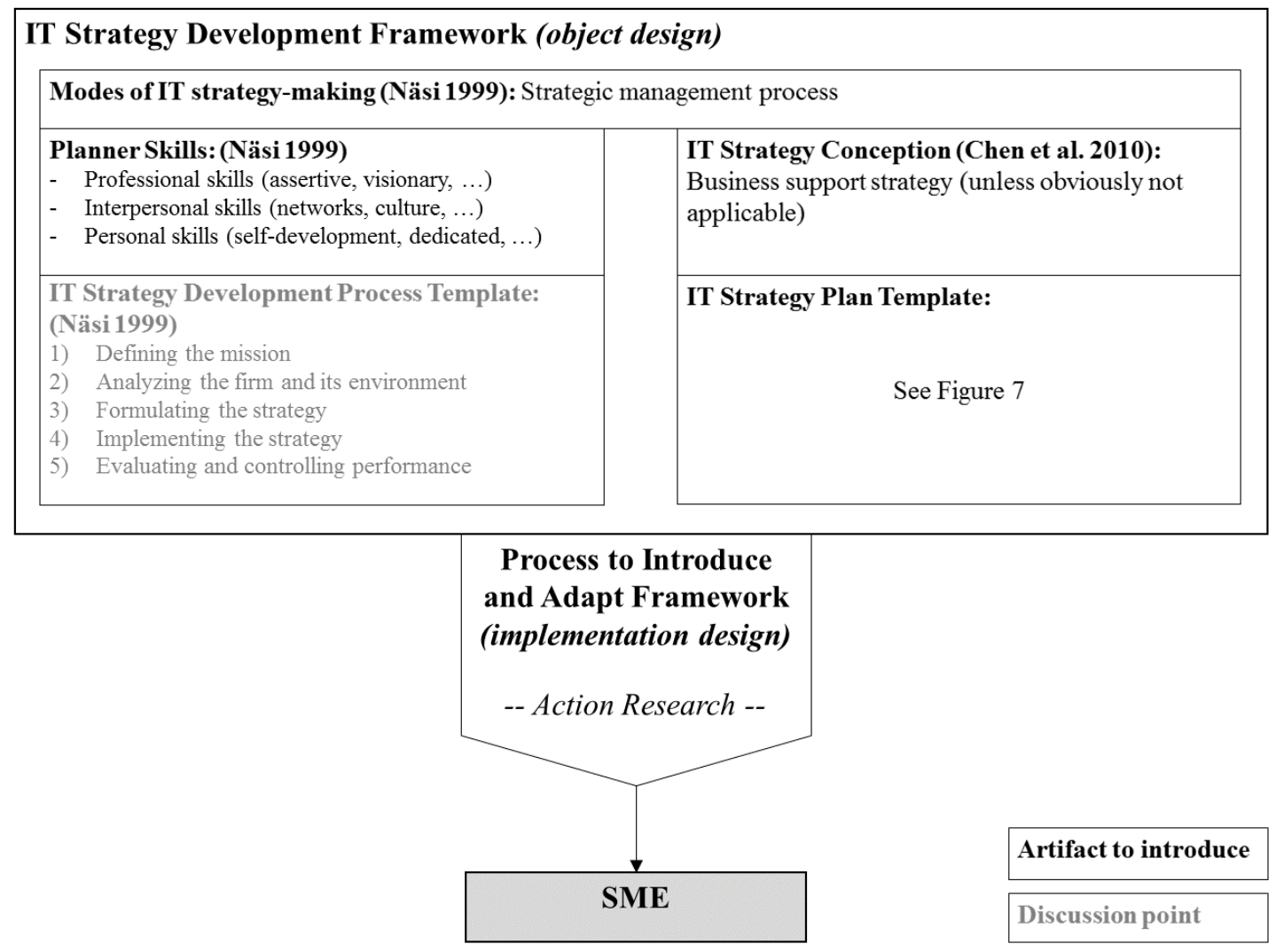

Fig. 6 The revised IT strategy development framework for SMEs

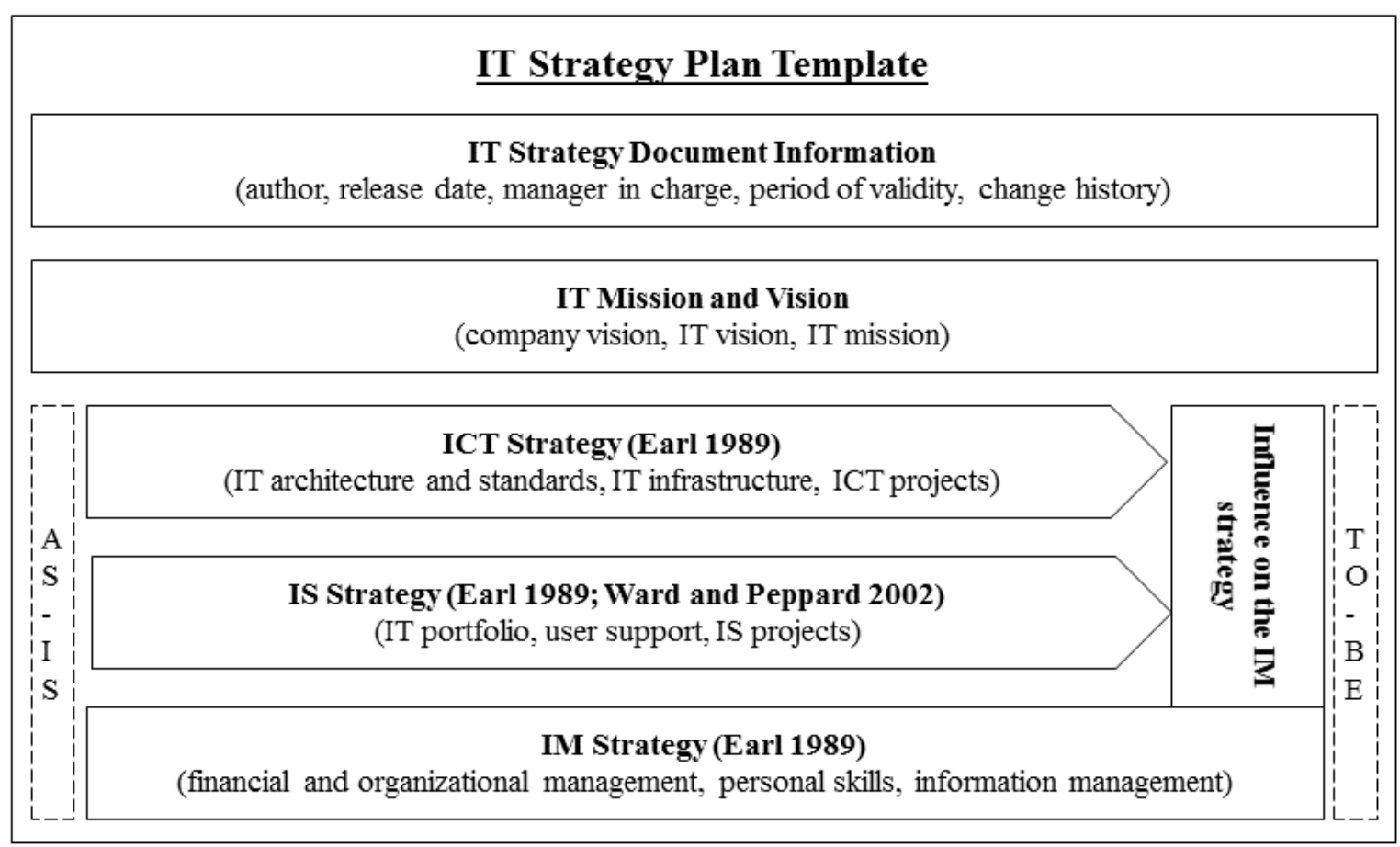

Fig. 7 The refined elements of the IT strategy plan template 
To conclude this third cycle, we subjected this redesigned version of the IT strategy development framework to another evaluation session with the same director with whom we had previously worked. He regarded the increased structure, to which several sub-strategies (IS, IM, ICT) had been added, as overall useful to address and structure decision-relevant issues that had been lacking before. The redesign therefore increased $\mathrm{DO}_{2}$ 's fulfilment.

However, the director did not find the new structure entirely intuitive regarding its simplicity, but he understood the rationale after a brief explanation. In his view, this structure allowed for emphasizing the relevant aspects and for increasing the IT strategy's scope over time, while retaining the overall cohesion. The director also found that the structure fostered task delegation. He had been skeptical at first, since he had associated an increase in the structure with less scope for freedom and individuality. In this case, however, the structure helped cover more issues. He even saw increased room for individuality due to the possibility to focus on particular IT sub-strategies or particular elements within the IT sub-strategies while de-emphasizing others that are less relevant in a given situation. The redesign therefore also increased $\mathrm{DO}_{3}$ 's fulfilment with regards to flexibility and comprehensiveness.

Further, the director regarded the introduction of an introductory and a concluding section as providing the strategy designer with specific guidance and a specific course of action to develop the strategy. At the same time, he did not believe that the guidance was a strict one, which he regarded as positive (in terms of allowing individuality) and negative (no formal assurance of completeness). However, he criticized the need to cover the strategy designer's personal traits. He believed that while they were important, they should not be part of an "impersonal document." Conversely, he found that adding the IT function's high-level goals (vision \& mission) in a section prior to the actual strategy emphasized their importance and directed the three sub-strategies better.

He regarded an executive summary as standard and therefore also useful for working with the document. Ideally, the executive summary would provide the exact sum that developing the IT strategy would save the SME in IT expenditure. Hence, the executive summary would provide a financial rationale as a foundation for evaluating the IT strategy's effectiveness in measureable terms. From the director's perspective, this management-oriented section summarizing the key points would also allow the other three sections (IM, IS, ICT) to be as detailed as required to address the two audiences of the IT strategy plan: 1) the business and IT management and 2) the IT personnel. In respect of the section containing document information, he emphasized that version numbers, dates, and the names of the responsible persons not only aided traceability and transparency, but also formalized personal responsibility for the IT strategy's continuous development. While this would not guarantee a continuous strategy process, he did regard it as an important step in the right direction.

From an overall perspective, the director was skeptical about the redesigned strategy plan's overall level of comprehensiveness and complexity. This skepticism was especially strong, given that the IT's only important goal was "to keep the lights on." Consequently, the director believed that it was important not to require every section and subsection to be filled out. He also pointed out that the integration of the IT strategy into other planning documents would be feasible, but simultaneously admitted that this was "wishful thinking," especially in small enterprises. Nevertheless, he believed that designing and formalizing an IT strategy was a good first step towards establishing a more thorough planning process.

In sum, the evaluation showed that the redesigned artifact was a step in the right direction, since the majority of redesigns were regarded as useful to increase the director's understanding of the IT's strategic potential $\left(\mathrm{DO}_{1}\right)$ and his ability to make better-informed IT strategy decisions $\left(\mathrm{DO}_{2}\right)$ that ultimately would lead to increased firm performance. While the redesign reduced the IT strategy plan template's simplicity level, the added introduction and overall structure allowed for tailoring the actual detail level more specifically to the context and audience, and thus retained the workability of the previous design, while simultaneously increasing the comprehensiveness and the flexibility $\left(\mathrm{DO}_{3}\right)$.

\subsection{Cycle 4: Deriving an initial implementation design for future applications}

Based on our experiences during the action research approach to introduce the first two versions of the IT strategy development framework in a particular SME, we now propose generic steps on how to apply the IT strategy development framework shown in Figures 6 and 7 in a new SME. The design science research methodology for 
IT management artifacts on which we relied (Drechsler 2013) calls this an implementation design artifact (cf. Section 3.2). Following van Aken $(2004 ; 2007)$, it suggests undertaking an initial redesign of the artifacts one wants to introduce - in our case the IT strategy plan template and the IT strategy process - without the involvement of future artifact users, followed by a second, potentially more extensive, redesign phase with intensive artifact user involvement. We assume that an initial contact has been made with a suitable SME and agreement has been reached to develop a new IT strategy by using the framework. Figure 8 shows the subsequent steps. We did not evaluate this proposed artifact in a new application context, hence this cycle remains a partial one.

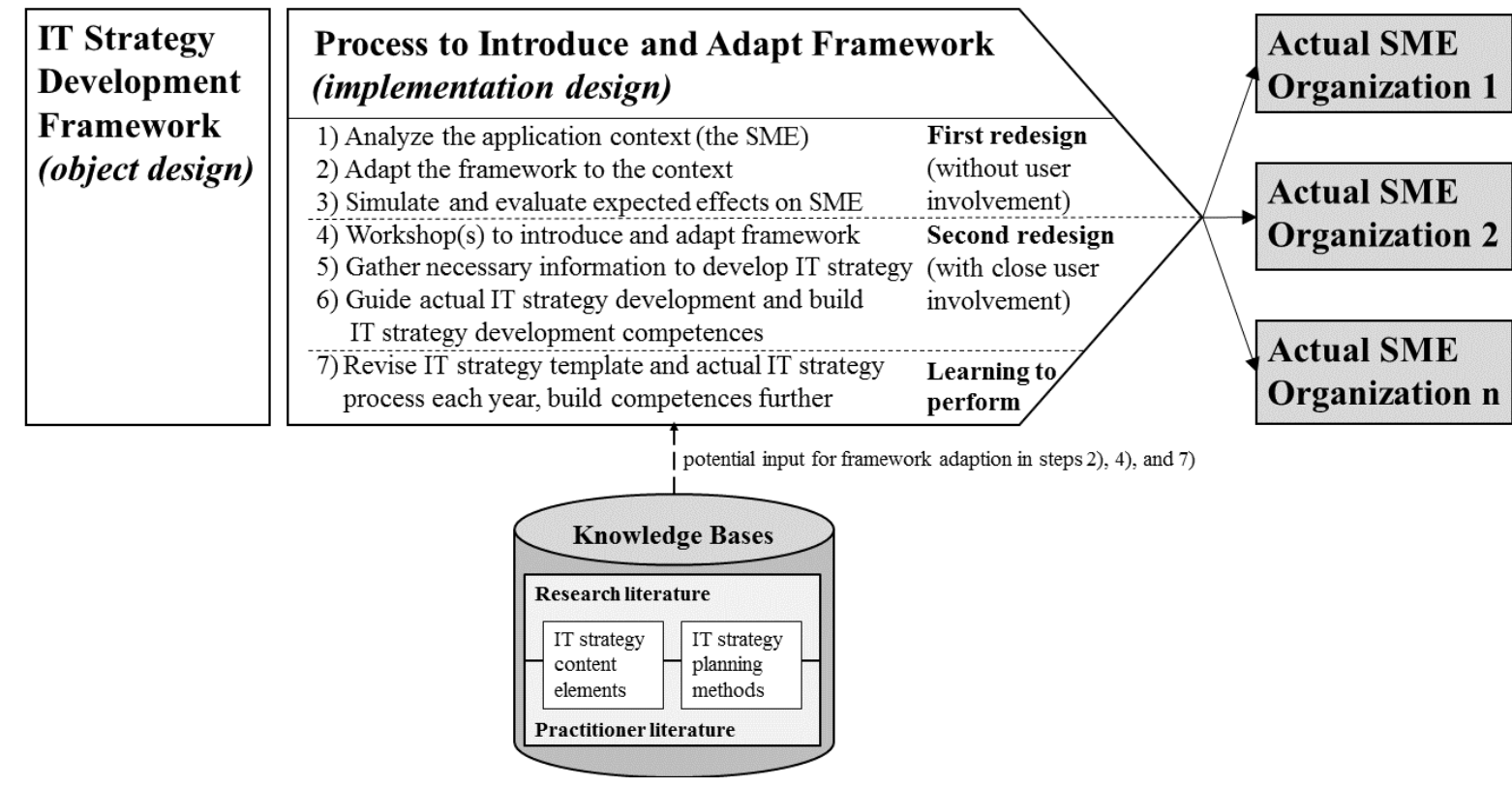

Fig. 8 Proposed steps for the implementation design to apply the IT strategy development framework to other SMEs

The implementation design's intended role as depicted in Figure 8 is to guide future applications of our IT strategy development framework. Every step needs to be tailored to the specific application context (a particular SME), which is why we leave it at the current abstraction level. Possible sources for the framework's adaption can be research and the practitioner-oriented literature as well as any existing IT strategies. While we recommend keeping the strategy content structure of Figure 7 (IT mission and vision, IM, IS, ICT strategy), the actual strategy content elements that are necessary, or useful, to consider within each partial strategy may vary. Here, Earl's (1989) or Ward and Peppard's (2002) seminal works (cf. Section 2.3) may serve as possible sources to carefully add new IT strategy content elements on, for example, the second level. The elements' names may also be altered to match established terminology within a particular SME. The same guidelines for adaption apply to the strategy planning process steps, or the planning methods used in each step. Here, should the need arise for more elaborate analysis and planning activities in a particular SME, one can draw, for instance, on Levy and Powell's (2000) or Duhan's (2007) works (cf. Section 2.4).

\section{Discussion}

In this section, we discuss the theoretical and practical contributions our IT strategy development framework makes, as well as contributions to social meta-artifact design in general that can be drawn from the framework's design and application. 


\subsection{Contributions of the IT strategy development framework for SMEs}

The IT strategy development framework we designed contributes to the IT strategy literature in the SME context in several ways. Viewed in Gregor and Hevner's (2013) contribution matrix, the IT strategy development framework artifact constitutes an "improvement." We designed a new solution for a known problem (poor resource use and a lack of IT strategies in SMEs), and evaluated the artifact in a typical small enterprise's application context over three full and one partial cycle of design, instantiation, and evaluation. We found that the framework's final version (Figures 6 and 7) fulfills the goal of attaining the "effectiveness" design objectives $\left(\mathrm{DO}_{1}\right.$ and $\mathrm{DO}_{2}$, cf. Section 3.2) as well as the "contextual adequacy" design objective $\left(\mathrm{DO}_{3}\right)$. In terms of effectiveness, the framework managed to increase the director's understanding of the IT's strategic potential, his ability to make well-informed IT strategy decisions, and to execute the IT strategy. In terms of contextual adequacy, the framework allowed a person without in-depth IT management competence to do so, provided a process that was easy to follow, and gave further guidance through an IT strategy document structure that balances comprehensiveness with a focus on the essential aspects for the specific context and that allows for flexibility on the second outline level.

We further argue that our framework is superior to the existing prescriptive knowledge - encompassing research and practitioner literature - on IT strategies for the SME context. In other words, we demonstrated the effective actionability (Kuechler and Vaishnavi 2011) of a particular combination of extant IT strategy knowledge (along with, in our first cycle, the lack of actionability of another combination of extant knowledge). We achieved this actionability by configuring and transforming existing descriptive and prescriptive knowledge into prescriptive knowledge tailored to the SME context (cf. Figures 5-7) that fulfilled our "effectiveness" and "contextual adequacy" design objectives. See Table 1 for a summary of the differences between our framework and the existing descriptive and prescriptive IT strategy development knowledge sources.

Table 1: Comparison between our framework and extant IT strategy development literature

\begin{tabular}{|l|l|l|l|l|}
\hline $\begin{array}{c}\text { Knowledge } \\
\text { source }\end{array}$ & Our framework & $\begin{array}{l}\text { IT strategy books } \\
\text { (Earl 1989; Ward } \\
\text { and Peppard 2002) }\end{array}$ & $\begin{array}{l}\text { SME-specific IT } \\
\text { strategy develop- } \\
\text { ment research } \\
\text { contributions } \\
\text { (Levy and Powell } \\
\text { 2000; Levy et al. } \\
\text { 2001; Duhan 2007) }\end{array}$ & $\begin{array}{l}\text { SME-specific IT } \\
\text { strategy develop- } \\
\text { ment practitioner } \\
\text { contribution - the } \\
\text { example of (Savin } \\
\text { 2004) }\end{array}$ \\
\hline $\begin{array}{l}\text { Spectrum of IT } \\
\text { strategy areas } \\
\text { (IS/IM/ICT) } \\
\text { covered }\end{array}$ & Comprehensive & Comprehensive & Selective & Limited \\
\hline $\begin{array}{l}\text { Extent of in-depth } \\
\text { coverage for IT } \\
\text { strategy areas }\end{array}$ & $\begin{array}{l}\text { Tailorable to the } \\
\text { application context } \\
\text { in question }\end{array}$ & $\begin{array}{l}\text { In-depth for each } \\
\text { area }\end{array}$ & $\begin{array}{l}\text { In-depth for the se- } \\
\text { lected areas }\end{array}$ & $\begin{array}{l}\text { Medium for the } \\
\text { ICT strategy (lack } \\
\text { of a to-be perspec- } \\
\text { tive), low for IS } \\
\text { and IM }\end{array}$ \\
\hline $\begin{array}{l}\text { Adaptability for } \\
\text { SME contexts }\end{array}$ & $\begin{array}{l}\text { High, guided by the } \\
\text { proposed imple- } \\
\text { mentation design } \\
\text { (Figure 8), as well } \\
\text { as the framework's } \\
\text { structure (Figures 6 } \\
\text { and 7) }\end{array}$ & $\begin{array}{l}\text { SME context not } \\
\text { explicitly covered }\end{array}$ & $\begin{array}{l}\text { Adaption not cov- } \\
\text { ered }\end{array}$ & $\begin{array}{l}\text { Adaption not cov- } \\
\text { ered }\end{array}$ \\
\hline $\begin{array}{l}\text { Actionability for } \\
\text { the SME context }\end{array}$ & $\begin{array}{l}\text { Medium (requires } \\
\text { adaption first) }\end{array}$ & Low & Low & $\begin{array}{l}\text { High (almost im- } \\
\text { mediate application }\end{array}$ \\
\hline
\end{tabular}




\begin{tabular}{|l|l|l|l|l|}
\hline & & & $\begin{array}{l}\text { possible, cf. Sec- } \\
\text { tion 4.1) }\end{array}$ \\
\hline $\begin{array}{l}\text { Overall suitability } \\
\text { for SME contexts }\end{array}$ & $\begin{array}{l}\text { High (the frame- } \\
\text { work's flexibility } \\
\text { should enable a fit } \\
\text { to differing con- } \\
\text { texts) }\end{array}$ & $\begin{array}{l}\text { Low (too compre- } \\
\text { hensive to apply) }\end{array}$ & $\begin{array}{l}\text { Low (too compre- } \\
\text { hensive to apply } \\
\text { despite limited } \\
\text { focus) }\end{array}$ & $\begin{array}{l}\text { Medium (lack of a } \\
\text { to-be perspective } \\
\text { and missing aspects } \\
\text { even for small con- } \\
\text { texts) }\end{array}$ \\
\hline
\end{tabular}

As practical contribution, the observed effects have the potential to lead to a better utilization of scarce - especially financial - resources in SMEs, which meets our research project's initial goal. While the absolute impact may be comparably small in a single SME, even a comparably small reduction in absolute IT expenses may well have a comparably large relative impact when compared to the overall budget. The same applies in an IT business value creation perspective: A comparably minor increase in business process effectiveness in absolute terms through improved information systems can still be significant in relative terms. And since the vast majority of enterprises are, in fact, SMEs (Australian Government 2014; European Commission 2014; U. S. Census Bureau 2014), the potential practical impact is large, even from an absolute perspective and assuming the framework's widespread adoption in the future.

Moving beyond the framework itself, we also contribute a more differentiated understanding of Chen et al.'s (2010) three IT strategy perspectives (business strategy implementation, effective IT asset allocation, shared understanding of the IT role across the organization, cf. Section 2.2) for an SME context without dedicated IT personnel and executives. Based on the study cited in the Introduction, such a context is typical of many SMEs when there is only an involuntary (operational level) IT manager at best. In contrast, Chen et al.'s (2010) third perspective assumes that there is already a (strategic) understanding of IT within an organization - although one that is usually not uniformly shared across an organization. In SMEs with only an involuntary strategic IT manager, such an understanding of IT does not necessarily exist. Therefore, Chen et al.'s (2010) third perspective is not directly applicable to these SME contexts. Here, our framework facilitates achieving such a strategic understanding of IT on the director level - especially if the involuntary (strategic) IT manager who uses our framework is a business executive, such as a director.

Along similar lines, we extend Chen et al.'s (2010) IT strategy conceptualization (Figure 1) for the SME context by highlighting the central role the strategic IT manager plays in initiating and following the IT strategy process, formulating an IT strategy, and driving the IT strategy's execution. We thus combine Chen et al.'s (2010) rather impersonal perspective on IT strategy with Näsi's (1999) leadership mode of IT strategy-making. Such a leadership-oriented perspective is particularly suited for the SME context, as indicated by the director we interviewed (cf. Section 4.2) and corroborated by Cragg et al. (2013) (cf. Section 2.4).

Furthermore, this paper offers one of the few instances of IS design science research not focused on developing an IT artifact, but a social (meta-)artifact aimed at a managerial audience in the IT management domain. The subsequent section covers the respective implications for the design science research discourse.

\subsection{Implications for social meta-artifact design in general}

In this section we reflect on more general implications for the design of future social-meta artifacts - referring to our IT strategy development framework as a sample instance of such a social meta-artifact (cf. Section 3.1).

At first glance, many paragraphs in the sections 4.1 to 4.4 concerning the four cycles seemingly deal with - from a research perspective -comparably minor details (the structure of the IT strategy template, the content of the introductory section, the executive summary, etc.) with little implications for the wider research discourse. However, our experience had shown that such details are crucial for the eventual adoption - and, hence, the utility of the social meta-artifact and the generated social artifacts (in the example above, the IT strategy plan and process) in the application context. These details are instances of, what Drechsler et al. (2016) call "artifact resonance". In a nutshell, resonance is a concept that addresses effective communication of research outcomes to practitioners (Gill 2010). For social meta-artifacts, achieving resonance thus means that practitioners adopt and 
subsequently use these meta-artifacts to make better-informed decisions or take better actions and to produce further artifacts that, in turn, provide the desired utility. Our experiences documented in the sections concerning the four cycles therefore contribute to the knowledge base on how to achieve social meta-artifact resonance in the SME context. Moreover, we recommend that future social meta-artifact design projects in all domains should pay attention to any details affecting future artifact resonance already during the initial artifact design and document them accordingly, in order to contribute to a growing body of artifact resonance knowledge.

Regarding our social meta-artifact's structure, the underlying descriptive knowledge of the corresponding domain context (IT strategy) led us to consider three distinct levels during the design: the content (the future IT strategy plan structure), the process (the corresponding IT strategy process), and the individual level (in terms of a strategic planner's traits). In turn, the "contextual adequacy" design objective, the emphasis on the content level in the prescriptive body of knowledge, and the feedback received in the cycles all played a role in guiding us to specifically emphasize the content level. In particular, the practitioner acknowledged that the process level is as important, but not crucially important, and he considered the integration of the traits into the strategy document of little use. However, the latter aspect conflicts with the possibility to achieve the same outcomes with social artifacts and meta-artifacts (management instruments), leadership (personally directing and intervening), and a combination of the two (as illustrated in Figure 3).

Consequently, we see this as an area for further research to transform related explanatory-oriented findings from the strategizing discourse (Jarratt and Stiles 2010) and the more general management and leadership discourse (Bennis 1989; Dionne et al. 2005; Ford and Harding 2007) into prescriptive knowledge for the design of social meta-artifacts with a managerial audience - within and also beyond the IT strategy development or SME contexts. Such further research also needs to pay attention to the different actors (researchers, managers) and the different artifact types (meta-artifacts, or artifacts generated by the meta-artifacts). As Figure 3 highlights, the management and leadership distinction also leads to particular challenges in evaluating social meta-artifacts as the evaluations need to address person-related impacts (leadership implications due to a cognitive impact), impersonal impacts (impacts on management systems and firm performance) and their effects on each other.

These deliberations furthermore raise the question whether the three levels "content", "process", and "individual / leader" can provide a useful basic structure for future social artifacts and/or meta-artifacts in general, or whether they are specific to IT strategy / IT management artifacts, or social artifacts, or meta-artifacts with a managerial audience. In turn, answering this question can be a step closer to answering the question of the basic nature of social meta-artifacts.

Since our initial artifact's application was undertaken with a more exploratory mindset, we also applied a novel combination of design and action research. This combination complements the development of a generic object design aimed at more than one context (cf. Iivari's (2015) strategy 1, Section 3.1) by means of action research to adapt and tailor this object design to a specific application context instead of following a more formalized implementation design. In turn, we synthesized our action research interventions into a proposal for an initial implementation design (Figure 8) for future applications. Considering two separate artifacts - the object design (the actual social meta-artifact) and the corresponding implementation design (in a sense, a social meta-meta-artifact) - also leads to the requirement to evaluate both in future research settings: the object design in terms of its utility to address real-world issues and the implementation design in terms of its utility to adapt and tailor the object design to a new application context.

\section{Limitations}

The instantiation of the IT strategy development framework in only a single application context is currently a major limitation. Future application contexts should include contexts of a similar nature, but also different ones, for example, SMEs of different sizes, or SMEs which rely heavily on outsourcing, or cloud computing, rather than their own IT infrastructure. Further, we did not evaluate the framework's impact on the actual firm performance (evaluation component $\mathrm{E}_{5}$ in Figure 3 ). This is partially due the low level of maturity of the accounting information system in the SME in question, but also due to the difficulty to isolate the framework's effects from others. Beyond the cognitive impact on the responsible manager, there are many other factors that can affect the 
initial organizational intervention constituting the introduction of an IT strategy development framework (downward block arrow "Adaption \& Introduction" in Figure 3). Likewise, many other factors can influence the triggered social artifact development (IT strategy process and plan) and many other factors can influence the impact that these developed social artifacts have on the organization (rightward block arrows in Figure 3). Our evaluation also does not cover whether the framework increased the quality of emergent strategic IT decisions (Mintzberg and Waters 1985). In addition, our proposal regarding the process with which to apply the framework in a new context (cf. Figure 8) is based on our action research process, but is as yet untested.

\section{Conclusion}

In this paper, we designed, applied, evaluated, and refined an IT strategy development framework for SMEs. Its instantiation allowed an SME's management to improve its understanding and ability to direct the IT function in the future and to utilize its IT resources for business purposes in a more structured and substantiated way. In a more abstract perspective, the IT strategy development framework constitutes a social meta-artifact that triggers organizational change and development processes to develop, or change, other social artifacts - such as an IT strategy process and an IT strategy plan.

In contrast to the assumption in the IT strategy literature that there is a strategic understanding of IT within an organization when addressing the shared understanding between the business and the IT, we determined that in our - not untypical - SME context there was no such strategic understanding of IT at all. Here, our framework not only helps achieving such an initial understanding, but its use also contributes to achieving a shared understanding of IT across the organization when a nominal business executive - for instance, a director - assumes the role of involuntary (strategic) IT manager by using the framework.

Thus, we are confident that - possibly with internal or external technical experts' relevant guidance - the IT strategy development framework will also be useful for voluntary or involuntary IT managers of other SMEs. We believe that the framework's utility in future SME contexts will improve their capabilities to direct the IT function and will not only provide increased transparency and understanding of IT's role in enhancing business processes, but also of how the information systems and the technology infrastructure collaborate. Both contribute to the SME's top management's abilities to make better-informed strategic IT decisions and to execute them.

Moreover, we do not see that using the IT strategy development framework in other application contexts has limitations as long as these contexts are rather typical of small enterprises outside the IT industry. Larger SMEs, or SMEs in which IT plays a more central role, can draw on existing complementary IT strategy content or process sources to conduct even more extensive IT strategy planning and expand the IT strategy plan accordingly. The same applies to a rapidly growing SME.

Finally, we once again wish to stress that we regard it as crucial that future users use the IT strategy plan template (Figure 7) and the entire IT strategy development framework (Figure 6) as blueprints to guide and inspire the IT strategy planning process's participant(s). The elements of an existing IT strategy can and should also be integrated into this framework. Depending on the existing IT strategy's maturity, it might be necessary to gather more detailed information about the actual as-is state of the ICT infrastructure, the information systems, or the existing information management processes, before the IT strategy makers can embark on their mission to develop intended future to-be states for each of the three IT strategy areas.

\section{References}

AMI Partners (2013) Involuntary IT Manager (IITM) Study.

Australian Government D of I (2014) Australian Small Business - Key Statistics and Analysis. http://www.innovation.gov.au/smallbusiness/keyfacts/Pages/Library\%20Card/AustralianSmallBusinessKeyStatisticsAndAnalysis.aspx. Accessed 4 Jun 2014

Bennis W (1989) Why Leaders Can’t Lead. Training \& Development Journal 43:35. 
Bergeron F, Raymond L, Rivard S (2001) Fit in strategic information technology management research: an empirical comparison of perspectives. Omega 29:125-142. doi: 10.1016/S0305-0483(00)00034-7

Bleistein SJ, Cox K, Verner J (2006a) Validating strategic alignment of organizational IT requirements using goal modeling and problem diagrams. Journal of Systems and Software 79:362-378.

Bleistein SJ, Cox K, Verner J, Phalp KT (2006b) B-SCP: A requirements analysis framework for validating strategic alignment of organizational IT based on strategy, context, and process. Information and software technology 48:846-868.

Bradshaw A, Cragg P, Pulakanam V (2012) Do IS consultants enhance IS competences in SMEs? Electronic Journal Information Systems Evaluation Volume 16:13-24.

Carr N (2003) IT doesn’t matter. Harvard Business Review 81:41-49.

Chao C-A, Chandra A (2012) Impact of owner's knowledge of information technology (IT) on strategic alignment and IT adoption in US small firms. Journal of Small Business and Enterprise Development 19:114-131.

Chen DQ, Mocker M, Preston DS, Teubner A (2010) Information systems strategy: reconceptualization, measurement, and implications. MIS Quarterly 34:233-259.

Cragg P, King M, Hussin H (2002) IT alignment and firm performance in small manufacturing firms. The Journal of Strategic Information Systems 11:109-132. doi: 10.1016/S0963-8687(02)00007-0

Cragg P, Mills A, Suraweera T (2013) The Influence of IT Management Sophistication and IT Support on IT Success in Small and Medium-Sized Enterprises. Journal of Small Business Management 51:617-636. doi: $10.1111 /$ jsbm.12001

Dionne SD, Yammarino FJ, Howell JP, Villa J (2005) Substitutes for leadership, or not. The Leadership Quarterly 16:169-193. doi: 10.1016/j.leaqua.2004.09.012

Drechsler A (2013) Design Science as Design of Social Systems - Implications for Information Systems Research. Journal of Information Technology Theory and Application 14:5-26.

Drechsler A, Hevner AR, Gill TG (2016) Beyond Rigor and Relevance: Exploring Artifact Resonance. In: 2016 49th Hawaii International Conference on System Sciences (HICSS). pp 4434-4443

Duhan S (2007) A capabilities based toolkit for strategic information systems planning in SMEs. International Journal of Information Management 27:352-367. doi: 10.1016/j.ijinfomgt.2007.03.001

Earl MJ (1989) Management strategies for information technology. Prentice Hall, New York

European Commission (2014) Fact and figures about the EU's Small and Medium Enterprise (SME). http://ec.europa.eu/enterprise/policies/sme/facts-figures-analysis/index_en.htm. Accessed 30 Jan 2014

Ford J, Harding N (2007) Move Over Management We Are All Leaders Now. Management Learning 38:475493. doi: $10.1177 / 1350507607083203$

Gill TG (2010) Informing Business: Research and Education on a Rugged Landscape. Informing Science Press, Santa Rosa, CA

Gill TG, Hevner AR (2013) A Fitness-Utility Model for Design Science Research. ACM Transactions on Management Information Systems 4:5:1-5:24. doi: 10.1145/2499962.2499963 
Gregor S, Hevner AR (2013) Positioning and presenting design science research for maximum impact. Management Information Systems Quarterly 37:337-355.

Gutierrez A, Orozco J, Serrano A (2009) Factors affecting IT and business alignment: a comparative study in SMEs and large organisations. Journal of Enterprise Information Management 22:197-211.

Hevner A (2007) A Three Cycle View of Design Science Research. Scandinavian Journal of Information Systems 19:87-92.

Hevner AR, March ST, Park J, Ram S (2004) Design Science in Information Systems Research. MIS Quarterly 28:75-105.

Hofmann J, Schmidt W (eds) (2007) Masterkurs IT-Management. Vieweg+Teubner Verlag, Wiesbaden

Iivari J (2003) The IS Core - VII: Towards Information Systems as a Science of Meta-Artifacts. Communications of the Association for Information Systems 12:Article 37.

Iivari J (2015) Distinguishing and contrasting two strategies for design science research. Eur J Inf Syst 24:107115. doi: $10.1057 /$ jis.2013.35

Iivari J (1986) Dimensions of information systems design: A framework for a long-range research program. Information Systems 11:185-197.

Iivari J, Maansaari J (1998) The usage of systems development methods: are we stuck to old practices? Information and software technology 40:501-510.

Jarratt D, Stiles D (2010) How are Methodologies and Tools Framing Managers' Strategizing Practice in Competitive Strategy Development? British Journal of Management 21:28-43. doi: 10.1111/j.14678551.2009.00665.x

Kuechler W, Vaishnavi V (2011) Promoting relevance in IS research: an informing system for design science research. Informing Science: the International Journal of an Emerging Transdiscipline 14:125-138.

Levy M, Powell P (2000) Information systems strategy for small and medium sized enterprises: an organisational perspective. The Journal of Strategic Information Systems 9:63-84. doi: 10.1016/S09638687(00)00028-7

Levy M, Powell P, Galliers R (1999) Assessing information systems strategy development frameworks in SMEs. Information \& Management 36:247-261.

Levy M, Powell P, Yetton P (2001) SMEs: aligning IS and the strategic context. Journal of Information Technology 16:133-144.

Lyles MA, Baird IS, Orris JB, Kuratko DF (1993) Formalized planning in small business: increasing strategic choices. Journal of Small Business Management 31:38-50.

Mandviwalla M (2015) Generating and Justifying Design Theory. Journal of the Association for Information Systems 16:314-344.

Maranto-Vargas D, Gómez-Tagle Rangel R (2007) Development of internal resources and capabilities as sources of differentiation of SME under increased global competition: A field study in Mexico. Technological Forecasting and Social Change 74:90-99. doi: 10.1016/j.techfore.2005.09.007

Mintzberg H, Waters JA (1985) Of strategies, deliberate and emergent. Strat Mgmt J 6:257-272. 
Näsi J (1999) Information systems and strategy design: The knowledge creation function in three modes of strategy-making. Decision Support Systems 26:137-149.

Nicolai AT, Seidl D (2010) That's Relevant! Different Forms of Practical Relevance in Management Science. Organization Studies 31:1257-1285. doi: 10.1177/0170840610374401

O'Connor RV, Coleman G (2009) Ignoring "Best Practice": Why Irish Software SMEs are Rejecting CMMI and ISO 9000. Australasian Journal of Information Systems. doi: 10.3127/ajis.v16i1.557

Olugbode M, Richards R, Biss T (2007) The role of information technology in achieving the organisation's strategic development goals: A case study. Information Systems 32:641-648. doi: 10.1016/j.is.2006.04.001

Pandza K, Thorpe R (2010) Management as Design, but What Kind of Design? An Appraisal of the Design Science Analogy for Management. British Journal of Management 21:171-186. doi: 10.1111/j.14678551.2008.00623.x

Peffers K, Tuunanen T, Rothenberger MA, Chatterjee S (2007) A Design Science Research Methodology for Information Systems Research. Journal of Management Information Systems 24:45-77.

Pries-Heje J, Baskerville R, Venable J (2008) Strategies for Design Science Research Evaluation. In: Proceedings of the ECIS 2008 conference, Galway, Ireland.

Proudlock M (1999) IT adoption strategies: best practice guidelines for professional SMEs. Journal of Small Business and Enterprise Development 6:240-252.

Rogers EM (2003) Diffusion of innovations. Free Press, New York

Samavi R, Yu E, Topaloglou T (2008) Strategic reasoning about business models: a conceptual modeling approach. Inf Syst E-Bus Manage 7:171-198. doi: 10.1007/s10257-008-0079-z

Savin JM (2004) Information technology strategy - managing the dark side. Handbook of Business Strategy 5:293-298. doi: http://dx.doi.org/10.1108/10775730410494134

Sein MK, Henfridsson O, Purao S, et al (2011) Action Design Research. MIS Quarterly 35:37-56.

Sexton DL, Van Auken P (1985) A Longitudinal Study of Small Business Strategic Planning. Journal of Small Business Management 23:7-15.

Simon HA (1996) The Sciences of the Artificial, 3. Aufl. MIT Press, Cambridge, Massachusetts

Singh RK, Garg SK, Deshmukh SG (2008) Strategy development by SMEs for competitiveness: a review. Benchmarking 15:525-547. doi: 10.1108/14635770810903132

U. S. Census Bureau (2014) Statistics about Business Size (including Small Business) from the U.S. Census Bureau. http://www.census.gov/econ/smallbus.html. Accessed 30 Jan 2014

van Aken JE (2004) Management Research Based on the Paradigm of the Design Sciences: The Quest for FieldTested and Grounded Technological Rules. Journal of Management Studies 41:219-246. doi: 10.1111/j.1467-6486.2004.00430.x

van Aken JE (2005) Management Research as a Design Science: Articulating the Research Products of Mode 2 Knowledge Production in Management. British Journal of Management 16:19-36. doi: Article

van Aken JE (2007) Design Science and Organization Development Interventions. Journal of Applied Behavioral Science 43:67-88. doi: Article 
Venkatraman N, Henderson JC, Oldach S (1993) Continuous strategic alignment: Exploiting information technology capabilities for competitive success. European Management Journal 11:139-149. doi:

10.1016/0263-2373(93)90037-I

Ward J, Peppard J (2002) Strategic planning for information systems. J. Wiley, Chichester, West Sussex, England; New York

Welsh JA, White JF (1982) A Small Business is not a Little Big Business. Harvard Business Review 59:18-33. 